\title{
Development of Glass Phosphor against Anti-Crone Ceramics
}

\author{
Masaki Fujikawa ${ }^{\mathrm{a},{ }^{*}}$, Mariko Hara ${ }^{\mathrm{a}}$, Yusuke Ozakia, and Shingo Fuchi ${ }^{\mathrm{b}}$ \\ ${ }^{\text {a} K o g a k u i n ~ U n i v e r s i t y, ~ 1-24-2, ~ N i s h i-S h i n j y u k u ~ 182-0022, ~ J a p a n ~}$ \\ ${ }^{\mathrm{b} A o y a m a}$ Gakuin University, 5-10-1, Fuchinobe 252-5258, Japan \\ *Corresponding Author: claveciniste@yahoo.co.jp
}

\begin{abstract}
Multi-modal artifact metrics as an anti-counterfeiting technique is created based on the concept of multi-modal biometrics. Giving two or more characteristic information to the artifact can improve the certainty of authenticity and increase counterfeiting difficulty. This study aims to develop a glass phosphor that gives two optical feature information, i.e., distribution of emission spectrum and emission intensity, to ceramic products. This phosphor emits visible and near-infrared light through optical excitation and wavelength of near-infrared light. It also demonstrates different distribution of emission spectrum and emission intensity at each observation point on the material. By welding a small amount of phosphor powder onto the ceramic surface, the authenticity and difficulty of counterfeiting could be enhanced in comparison with the existing method we proposed. In our previous study, the abovementioned phosphor was developed by adding two types of rare earth oxides. However, it was not transparent and was yellow in color. Herein, we introduce a new glass phosphor which is transparent and pale in color, and achieves high emission intensity by changing the composition of the base glass material.
\end{abstract}

Keywords: glass phosphor, multimodal artifact metrics, distribution of emission spectrum and emission intensity.

\section{Introduction}

\subsection{Background and Target}

Ceramic products made by prestigious as well as well-known potteries or brand holders are highly popular and can be sold at a high price as high-quality accessories. Counterfeiters manufacture imitations of these products ${ }^{(1)}$ and sell them to people with low appraisal skills. The existence of copied ceramic products cannot be overlooked, because the distribution of counterfeit products not only violates the intellectual property rights of potteries and brand holders but cost them a share of income that they could have otherwise obtained. Besides, financial and psychological damages can also be made to consumers who purchase them unknowingly.

We have developed a technique that can verify the product's authenticity mechanically with high accuracy to limit people with low appraisal skills to purchase imitations unknowingly. This technique is defined as the artifact metrics (2) because it makes it difficult for forgers to manufacture the genuine product's clone. In our previous research ${ }^{(3)}$, we focused on a transparent glass phosphor that emits one peak wavelength in response to infrared (IR) light excitement. We also proposed a technique to weld small amount of phosphor powder onto the surface of products. It has three features as shown as follows:

(1) The powder has low toxicity and does not affect the product's colors/patterns.

(2) The characteristic information (emission intensity) used for verification of authenticity can be extracted from each product in a non-contact manner.

(3) It is difficult to manufacture clones using the extracted characteristic information from genuine products.

We also have proposed a concept called the multi-modal artifact metrics hinted by multi-modal biometrics ${ }^{(4)}$ to enhance the accuracy of authenticity and difficulty of counterfeiting by adding over another characteristic information to the artifact. As a countermeasure of counterfeiting valuable cards (such as credit cards), we proposed a method of adding both sheet resistance (as characteristic electrical information) and phosphor emission (as optical feature information) to 
synthetic resin cards. ${ }^{(5)}$

To date, we have been trying an approach of applying the multimodal artifact metrics to ceramics and extracting several characteristic optical information from them. In this paper, we describe the development of new glass phosphor for this optical approach. By welding small amount of phosphor on the surface of such ceramic products, the resultant film is expected to give two optical feature information (hue and emission intensity). Therefore, the accuracy of authenticity and difficulty of counterfeiting could be enhanced compared to our previous study findings ${ }^{(3)}$. Details will be described in Section 2.

This paper is organized as follows: In the remaining part of Section 1, the artifact metrics and multi-modal artifact metrics are outlined. Section 2 provides an outline of our idea while the making prototype glass phosphor is described in Section 3. Considerations based on the experimental results are then given in Section 4, while Section 5 finally draws the conclusions.

\subsection{Outline of the Artifact Metrics}

Basically, the concept of artifact metrics is similar to biometrics. Each artifact has unique characteristic information. Artifact's authenticity can be verified by extracting characteristic information from target artifact and comparing it with the registered feature information. The difficulty of counterfeiting is established based on technological evidence, by which the characteristic information held by the genuine product is difficult to copy. This characteristic information is formed spontaneously and randomly during the manufacturing process, and cannot be formed intentionally even by manufacturers of genuine products. Hence, the difficulty of counterfeiting cannot be decreased if the mechanism of forming characteristic information is accessible to the public.

Microscopically, each artifact has different characteristic information, such as tiny roughness and color shade. However, specifying the area photographed by registration phase and the area shot by verification phase is time consuming, because the area observed using a microscope is quite small ${ }^{(3)}$. Hence, in artifact metrics, a method is utilized to form unique and easy-to-extract characteristic information in the artifact. Specifically, additives having physical features are added during the manufacturing process. The particles are distributed randomly and fixed in the artifact. The distribution degree reflects the characteristic information. Sensing devices which can extract the physical features of additives are used
Table 1. Physical characteristics \& extracted information.

\begin{tabular}{|c|l|}
\hline $\begin{array}{c}\text { Physical } \\
\text { characteristics }\end{array}$ & \multicolumn{1}{|c|}{ Extracted feature information } \\
\hline $\begin{array}{c}\text { Optical } \\
\text { characteristics }\end{array}$ & $\begin{array}{l}\text { Particles' optical characteristics (reflection, } \\
\text { transmission, infraction, and fluorescence) and their } \\
\text { degree of distribution reflect the characteristic } \\
\text { information, which is extracted by sensors that can } \\
\text { detect light intensity. }\end{array}$ \\
\hline $\begin{array}{c}\text { Magnetic } \\
\text { characteristics }\end{array}$ & $\begin{array}{l}\text { Particles' magnetic characteristics (attraction and } \\
\text { repulsive force) and their degree of distribution } \\
\text { reflect the characteristic information, which is } \\
\text { extracted by sensors that can detect a change in } \\
\text { magnetism. }\end{array}$ \\
\hline $\begin{array}{c}\text { Electrical } \\
\text { characteristics }\end{array}$ & $\begin{array}{l}\text { Particles' electric characteristics (electrical charge) } \\
\text { and the degree of distribution reflect the } \\
\text { characteristic information, which is extracted by } \\
\text { sensors that can detect the quantity of electric } \\
\text { charge. }\end{array}$ \\
\hline $\begin{array}{l}\text { Vibration } \\
\text { characteristics }\end{array}$ & $\begin{array}{l}\text { Particles' vibration characteristics (sonic waves) and } \\
\text { the degree of distribution reflect the characteristic } \\
\text { information, which is extracted by sensors that can } \\
\text { detect sonic waves. }\end{array}$ \\
\hline
\end{tabular}

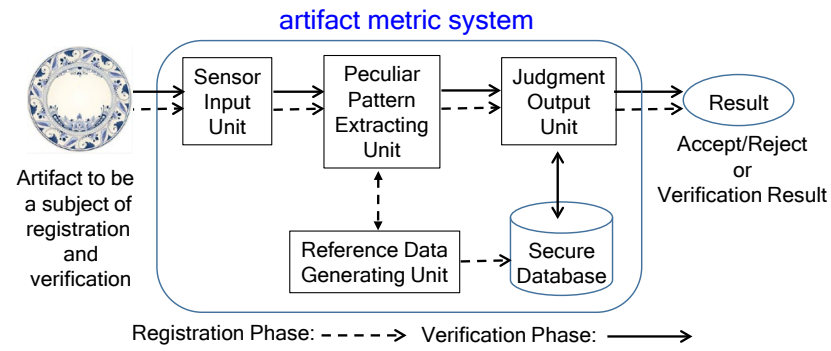

Fig. 1. Overview of the artifact metric system.

when extracting characteristic information.

Table 1 shows the physical characteristics of materials added in the manufacturing process and the characteristic information extracted from them. Fig. 1 shows an overview of the system (artifact metric system) that uses artifact metrics. Two phases are almost the same as those of biometrics. In this system, characteristic information that is extracted from each artifact before it is shipped is stored in Secure Database. To verify the authenticity of an artifact, the system extracts the characteristic information from it and compares the information with that of the registered feature in Secure Database.

\subsection{Outline of the Multimodal Artifact Metrics}

Characteristic information extracted from artifacts can be changed, depending on environmental circumstances during extraction (such as temperature, humidity, and position of artifacts relative to sensing devices). However, even in such situations, the artifact metric system should be able to verify authenticity stably and correctly, using the strong correlation between the characteristic information registered in the database and the information features 
extracted during verification.

There is an approach to increase the number of characteristic information to find strong correlations between both registered and extracted information ${ }^{(3)}$. This approach is named "multi-modal artifact metrics (5)" because various characteristic information is used. This approach can be further categorized into two methods. The first one (approach 1) focuses on additives having one physical feature and extracts two or more types of characteristic information from the artifact. For example, in our previous paper ${ }^{(3)}$, especially in the "consideration" section, we focused on the optical feature of glass phosphor and proposed another idea to utilize two distributions (emission spectrum and emission intensity distribution) as the characteristic information. These distributions can be reflected by the particle size and degree of dispersion of glass phosphor welded on the surface of ceramic products.

The second method (approach 2) adds two or more additives having different physical features to the artifact, from which two or more types of characteristic information can be extracted. For example, in our previous study ${ }^{(5)}$, we proposed an idea of creating thin films inside synthetic resin cards (used for valuable cards) using a conductive polymer paint having electrical features, and infrared up-conversion phosphor powder having optical features. This method utilizes emission intensity and sheet resistance which reflect the size and dispersion of phosphor particles in thin films. This information could be used as the characteristic information.

The differences of both methods are listed in Table. 2 . Although approach 1 is limited by the amount of characteristic information extracted from the artifact, it could reduce the quantity of materials for the total amount of artifacts. Approach 2 could increase more characteristic information than the former one. However, it could also affect the artifact's moldability and physical strength, because the material quantity for the total amount of artifacts increases.

Table 2. Comparison between two approaches.

\begin{tabular}{|c|l|l|}
\hline \multirow{3}{*}{ Advantages } & $\begin{array}{l}\text { Approach 1 } \\
\text { Low probability of } \\
\text { affecting the artifact's } \\
\text { moldability and physical } \\
\text { strength. }\end{array}$ & $\begin{array}{l}\text { Apcreases the number of } \\
\text { extractable feature } \\
\text { information. }\end{array}$ \\
\hline Drawbacks & $\begin{array}{l}\text { Limited with regard to } \\
\text { the number of extractable } \\
\text { feature information. }\end{array}$ & $\begin{array}{l}\text { High probability of } \\
\text { affecting the artifact's } \\
\text { moldability and physical } \\
\text { strength. }\end{array}$ \\
\hline
\end{tabular}

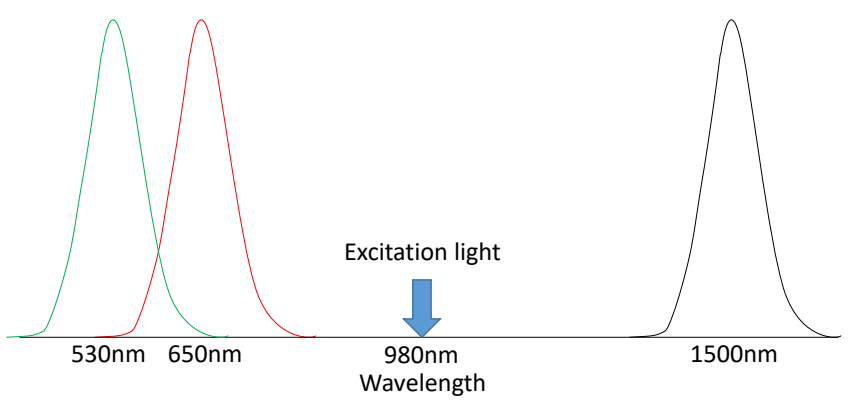

Fig. 2. Emission peak wavelengths and optical excitation.
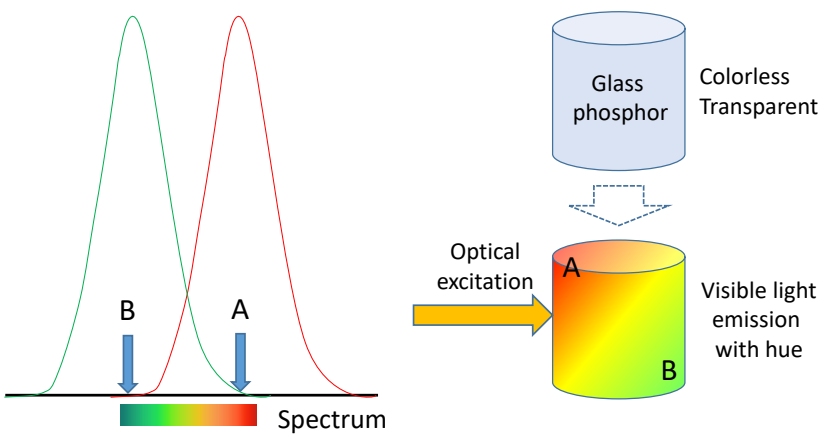

Fig. 3. Visible light emission (Vis. range).
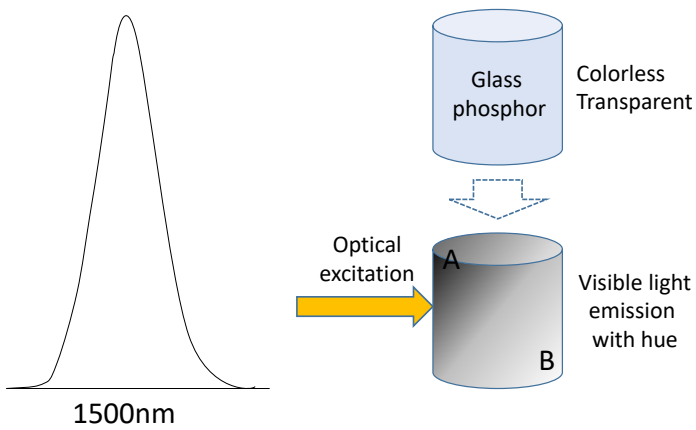

Fig. 4. Infrared light emission (NIR range).

\section{Approach}

Unlike the phosphor described in our previous study ${ }^{(3)}$, the novel glass phosphor shows different light emissions and intensity based on the locations where the excitation light is irradiated. Fig. 2 shows an image of emission peak wavelengths of the glass phosphor, Fig. 3 shows an image of emission in visible light band, and Fig. 4 shows an image of emission in near infrared light band, respectively. As shown in Fig. 3, red color could be observed at observation point $\mathrm{A}$, while green color could be observed at observation point $\mathrm{B}$. The yellow color means that red and green light are emitted at that point. In Fig. 4, the emission with peak wavelength of $1500 \mathrm{~nm}$ could be observed, so that the difference of the distribution of emission spectrum and emission intensity described in the previous study ${ }^{(3)}$ could 
also be observed. The difference of these distributions based on the observation point occurs because of the distance between ions and the ratio difference of the number of ions from multiple rare earth oxides contained in glass phosphor.

Similar to our previous study ${ }^{(3)}$, we chose to weld the glass phosphor powder onto the surface of ceramic products. Two types of feature information, namely, emission spectrum distributions (hue) and emission intensity distributions, could be extracted by photograph, when the unevenness of distance between ions and the number of ions were retained in the powder.

Generally, the phosphor which requires infrared as light source was developed with the aim of having one sharp peak wavelength (or single color), which exhibits one-peak wavelength in the visible light band or the near infrared light band. Because so far, developing a phosphor with emission from two light bands by changing the ratio of rare earth oxides has been rarely reported, our approach seems to be novel. An approach exists for developing the down-conversion glass phosphor having the same features, which use an ultraviolet (UV) light source. However, UV light could affect the colors/pattern of the ceramic during the irradiation on the surface of ceramic products. Hence, in this study, we developed the phosphor which utilizes an infrared light source.

\section{Making Prototype Base Glass Material}

Glass phosphor comprises base glass material and rare earth oxide. We previously reported that the glass phosphor could be obtained by adding two types of rare earth oxides (6). However, it was yellow in color and was not transparent. In this section, we reconsider the composition of the base glass material, especially the modified oxide, to make glass phosphor transparent and rare in color. This assists glass phosphor to be characterized with the different emission distribution spectrum and emission intensity. We also search appropriate ratio of rare earth oxide for newly created base glass material.

Table. 3 Candidate composition of base glass material.

\begin{tabular}{|c|c||c|c|}
\hline No. & Composition & No. & Composition \\
\hline 1 & $\mathrm{GeO}_{2}$ & 4 & $\mathrm{GeO}_{2}-\mathrm{Sb}_{2} \mathrm{O}_{3}$ \\
\hline 2 & $\mathrm{TeO}_{2}$ & 5 & $\mathrm{GeO}_{2}-\mathrm{MoO}_{3}$ \\
\hline 3 & $\mathrm{Sb}_{2} \mathrm{O}_{3}$ & 6 & $\mathrm{GeO}_{2}-\mathrm{Li}_{2} \mathrm{CO}_{3}$ \\
\hline
\end{tabular}

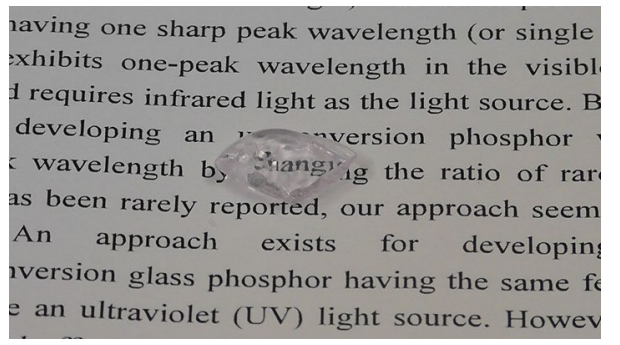

Fig. 5 Appearance of base glass material $\left(75 \mathrm{GeO}_{2}-25 \mathrm{Li}_{2} \mathrm{CO}_{3}\right)$

\subsection{Improvement of Transparency}

We reconsidered the modified oxide for the base glass material and satisfied conditions are listed as follows:

(1) Nonpoisonous/deleterious

(2) Powder color is whitish (in general, scattered light causes the color).

(3) The melting point is $1400^{\circ} \mathrm{C}$ or below. The temperature in furnace is also the same. For example, glaze firing for porcelain requires high temperature between 1200 and $1300^{\circ} \mathrm{C}$ ).

We selected five candidate materials based on the above conditions: $\mathrm{GeO}_{2}, \mathrm{TeO}_{2}, \mathrm{Sb}_{2} \mathrm{O}_{3}, \mathrm{MoO}_{3}$, and $\mathrm{Li}_{2} \mathrm{CO}_{3}$. As shown in Table 3, we arrange six candidate compositions based on their base glass material and prototypes. We adjusted the amount of each material to create transparent and colorless prototype while observing its appearance. Finally, we found that a prototype composition No. 6 of $75 \mathrm{GeO}_{2}-25 \mathrm{Li}_{2} \mathrm{CO}_{3}$ was colorless with high transparent appearance, as shown in Fig. 5. However, we had a concern that the base glass material could become clouded after a certain period due to the moisture absorbing ability of $\mathrm{Li}_{2} \mathrm{CO}_{3}$. Thus, we conducted an observation to verify whether it could become clouded or not within seven months. As the base glass material did not show any cloudy appearance after observation, it could be used as a material for making glass phosphor.

\subsection{Ratio of Rare Earth Oxide}

To create a new glass phosphor described in Section 2 (see Figs. 2 to 4), we tried to find an appropriate ratio of rare earth oxides to be added to the base glass material $\left(75 \mathrm{GeO}_{2}-25 \mathrm{Li}_{2} \mathrm{CO}_{3}\right)$. We selected $\mathrm{Er}_{2} \mathrm{O}_{3}$ and $\mathrm{Yb}_{2} \mathrm{O}_{3}$ because the former emitted red and green light in the visible light band and infrared light, whereas the latter transmitted excitation energy to Erbium ions while absorbing infrared light. 
Table. 4 Combination ratio of two rare earth oxide.

\begin{tabular}{|c|c|c|c|c|c|c|c|}
\hline No. & 1 & 2 & 3 & 4 & 5 & 6 & 7 \\
\hline $\mathrm{Yb}_{2} \mathrm{O}_{3}$ & 1.0 & 2.0 & 3.0 & 3.0 & 4.0 & 5.0 & 5.0 \\
\hline $\mathrm{Er}_{2} \mathrm{O}_{3}$ & 0.02 & 0.05 & 0.03 & 0.07 & 0.06 & 0.07 & 0.08 \\
\hline
\end{tabular}

laving one sharp peak wavelength (or single
exhibits one-peak wavelength in the visibl
1 requires infrared light as the light source. B
developing an
wavelength b,
as been rarely reported, our approach seem
An approach exists for developin
iversion glass phosphor having the same ff
an ultraviolet (UV) light source. Howev

Fig. 6 Glass phosphor (erbium and ytterbium oxide were added to the base glass material, $75 \mathrm{GeO}_{2}-25 \mathrm{Li}_{2} \mathrm{CO}_{3}$ )

The glass phosphor can be made by putting reagents (Ytterbium, Erbium, and base glass material) into a crucible, melting them in a furnace (about 1230 degrees Celsius for several tens of minutes) and cooling it rapidly after extracting from the furnace. These compositions could generate points in the glass phosphor, where the numbers of Ytterbium and Erbium ions and the distance between them would be spontaneously uneven during the synthesis process. This phenomenon could provide different emission spectra (hue) and emission intensity at varied observation points. The emission spectra (hue) can be changed by the distance between ions and the ratio of the numbers of Ytterbium and Erbium ions, while emission intensity in visible and infrared light band was controlled by the number of Erbium ions.

In our experiment, we made candidates of glass phosphor by changing the weight of each $\mathrm{Er}_{2} \mathrm{O}_{3}$ and $\mathrm{Yb}_{2} \mathrm{O}_{3}$, while the mass of the glass phosphor was fixed as 5 grams. Table 4 shows the candidate combination ratios of two rare earth oxides. These ratios were derived from our previous study ${ }^{(6)}$, where the combination of $5.0 \quad \mathrm{Yb}_{2} \mathrm{O}_{3}$ and 0.08 $\mathrm{Er}_{2} \mathrm{O}_{3}$ provided different emission spectra (hue) and emission intensity. Although the combination of Nos. 6 and 7 caused cloudiness in each candidate phosphor, the others provided two peak wavelengths in the visible light band and one-peak wavelength at $1500 \mathrm{~nm}$ in the infrared light band with the transparent appearance. The reason for the presence of the cloudiness in the glass phosphor could be associated with an increase in the amount of $\mathrm{Yb}_{2} \mathrm{O}_{3}$ as it was unable to melt completely in the furnace. Of these candidates, No. $4\left(3.0 \mathrm{Yb}_{2} \mathrm{O}_{3}\right.$ and $0.07 \mathrm{Er}_{2} \mathrm{O}_{3}$ ) provided the strongest emission peaks while receiving excitation light of

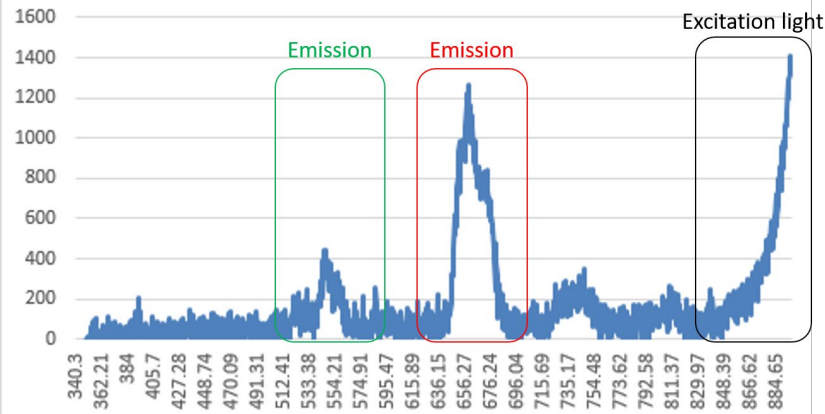

Fig. 7 Emission spectrum in the visible light band.

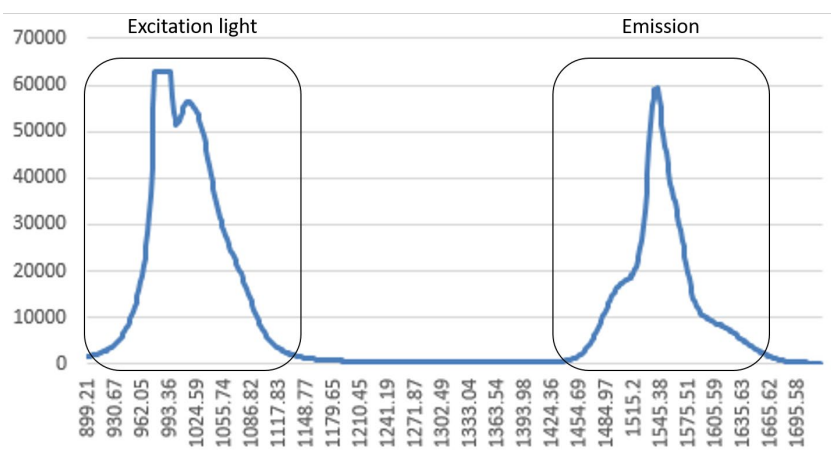

Fig. 8 Emission spectrum in the near infrared light band.

$980 \mathrm{~nm}$. Fig. 6 shows the appearance of the glass phosphor, and Figs. 7 and 8 show the emission spectrum of the visible light and the infrared light band, respectively. Our intended emissions were observed in each light band.

\section{Considerations}

\subsection{Safety of Glass Phosphor}

While small amounts of rare earth elements are used to produce the glass phosphor, no apparent toxicity can be observed from the rare earth elements ${ }^{(7)}$. Materials of the supporting glass (oxide glasses of boron oxide system, phosphoric acid system, and boron anhydrate system) are stable oxides that are incombustible and insoluble. Their toxicities are very low. For example, lead glass is contained in high quality transparent glass called crystal glass. Such glass is used as tableware and brings a serious safety concern. Furthermore, glass phosphor that is the compound between rare earth elements and the supporting glass is an incombustible and insoluble oxide glass. As mentioned above, glass phosphor added to the artifact is less risky in affecting human health and environment. However, care should be taken to prevent inhalation and adhesion of the particles to naked eyes in handling the powders. 
Table 5. Comparison between glass phosphor and others.

\begin{tabular}{ll}
\hline \hline \multicolumn{1}{c}{ Material } & \multicolumn{1}{c}{ Consideration } \\
\hline $\begin{array}{l}\text { Transparent magnetic } \\
\text { substance }\end{array}$ & $\begin{array}{l}\text { The magnetizing process is required after } \\
\text { firing because the magnetic force wanes at } \\
\text { high heat during firing }{ }^{(8)} \text {. }\end{array}$ \\
\hline $\begin{array}{l}\text { Transparent conductive } \\
\text { material }\end{array}$ & $\begin{array}{l}\text { For example, ITO (Indium Tin Oxide) } \\
\text { loses transparency above } 600 \text { degrees C } \\
\text { during firing }{ }^{(9)} \text {. }\end{array}$ \\
\hline $\begin{array}{l}\text { Quartz with rare earth } \\
\text { oxide }\end{array}$ & $\begin{array}{l}\text { It cannot melt 1200 to 1300 degrees C } \\
\text { during firing. It melts above 1550 degrees } \\
\text { C. }\end{array}$ \\
\hline Glass phosphor & $\begin{array}{l}\text { It melts 1200 to 1300 degrees C and } \\
\text { doesn't lose its function (transparency and }\end{array}$ \\
IR emission) after firing.
\end{tabular}

Table 6. Reagent's name, the amount used for making the glass phosphor (No.4) described in Section 3.2, and the retail price per gram.

\begin{tabular}{c|c|c}
\hline \hline Name & The amount used & Retail price / Gram \\
\hline $\mathrm{Yb}_{2} \mathrm{O}_{3}$ & 0.62 gram & $2.65 \mathrm{USD} /$ gram \\
\hline $\mathrm{Er}_{2} \mathrm{O}_{3}$ & 0.01 gram & $3.60 \mathrm{USD} /$ gram \\
\hline $\mathrm{GeO}_{2}$ & 3.99 gram & $19.38 \mathrm{USD} /$ gram \\
\hline $\mathrm{Li}_{2} \mathrm{CO}_{3}$ & 0.94 gram & $0.63 \mathrm{USD} /$ gram \\
\hline
\end{tabular}

\subsection{Advantages of Glass Phosphor}

Authors has been pursuing developing colorless and transparent glass phosphor. Although some transparent materials exist, each candidate has application problems to for the porcelain products (See Table. 5). This shows the advantage of glass phosphor for practical use.

\subsection{Cost of Glass Phosphor per Product}

Table. 6 shows the reagent's name, the amount used for making the glass phosphor (No.4), and the reagent's retail price per gram. Hence, the price of all reagents for making 5 grams of glass phosphor was 79.55 USD. In our previous study ${ }^{(3)}$, the average amount of glass phosphor powder welded onto the surface of one pottery plate was $\sim 0.05$ grams; therefore, the price of all the reagents for one plate would be $\sim 0.79$ USD. The price for the welded glass phosphor powder was the same or less than the price of one RFID tag of the same square measure, i.e., between 0.72 and 1.09 USD. Although pasting an RFID tag onto the surface of each product would impair its colors/patterns, welding a small amount of glass phosphor would not. Hence, the glass phosphor could be one of the security products with relatively cheap and design impairment-free features.

The cost described above was the price of making 5 grams of the glass phosphor in the experimental environment. We used easily available rare earth oxides in our experiment, and the weight ratio to the base glass material was $\sim 13 \%$. In practical and commercial use, the mass production of glass phosphor is possible and the cost of manufacturing the glass phosphor could be less than the abovementioned price.

\section{Conclusions}

We developed the glass phosphor which shows two peak wavelengths in visible light band and one peak wavelength in infrared light band by optical excitation to apply the multi modal artifact metrics for ceramic products. This phosphor could increase characteristic information in the products. Two optical feature information (hue and emission intensity) could be extracted from any observation point by welding glass phosphor powder onto the ceramic product during manufacturing process. The authenticity could be verified using registered images stored in the artifact metric system because two characteristic information was recorded as images in verification phase.

To create a colorless and transparent glass phosphor, we selected candidate modified oxides for making base glass material. In the composition of the material $75 \mathrm{GeO}_{2}-$ $25 \mathrm{Li}_{2} \mathrm{CO}_{3}$, the abovementioned features were present with no cloudiness during the follow-up observation. We also made the transparent glass phosphor with $3.0 \mathrm{Yb}_{2} \mathrm{O}_{3}$ and $0.07 \mathrm{Er}_{2} \mathrm{O}_{3}$. In future, we plan to weld the glass phosphor powder onto the surface of ceramic products and observe the light emission in both light bands.

\section{Acknowledgment}

This work was supported by JSPS KAKENHI Grant Number 18K11302 and The Kazuchika Okura Memorial Foundation.

\section{References}

(1) MeissenPorcelain.com. (July 2013). Meissen Porcelain Reproductions and Fakes. [Online]. Available: http://meissenporcelain.com/meissen-porcelain-reprod $\underline{\text { uctions/ }}$

(2) T. Matsumoto, M. Hoga, Y. Ohyagi, M. Ishikawa, M. Naruse, K. Hanaki, R. Suzuki, D. Sekiguchi, N. Tate, and M. Ohtsu, "Nano-artifact metrics based on random collapse of resist," Nature Science Reports 4, Article number 6142, pp. 1-5, 2012.

(3) M. Fujikawa, F. Oda, K. Moriyasu, S. Fuchi, and Y. Takeda, "Development of the New Artifact-metircs 
Technology for Valuable Pottery and Porcelain Products," Journal of Information Processing Society of Japan, vol. 55, no. 9, pp. 1992-2007, 2014.

(4) A. Ross and A. K. Jain, "Multimodal Biometrics: An overview," Proc. of 12th European Signal Processing Conference (EUSIPCO), pp. 1221-1224, 2004.

(5) M. Fujikawa, J. Kouki, and S. Fuchi, "A Study on the Multimodal Artifact Metrics and its Application Possibility (Application for Synthetic Resin Products)," The Japanese Journal of the Institute of Industrial Applications Engineers, vol. 5, No. 2, pp. 52-62, 2017.

(6) M. Fujikawa, M. Hara, and S. Fuchi, "A Study on Anti-Clone and Authenticity Verification Technique for Ceramics: Pilot testing of New Glass Phosphor with Two Optical Features," Proc. of the 6th IIAE International Conference on Intelligent Systems and Image Processing 2018, pp. 349-356, 2016.

(7) Y. Suzuki, "Popular science: Kidorui-no-hanashi". (Japanese text). Shokabo publishing Co., Ltd, pp. 144-146.

(8) E. J. Schwarz, "A Discussion of Thermal and Alternating Field Demagnetization Methods in the Estimation of Paleomagnetic Field Intensities," Journal of Geomagnetism and Geoelectricity, Vol. 21, No. 3, pp. 669-677, 1969

(9) K. Goto, T. Kawashima, and N. Tanabe, "Transparent Conductive Glass", Fujikura Technical Journal, pp. $57 / 61,2004$ 